\title{
EFECTO DE LOS CULTIVOS DE COBERTURA SOBRE LAS PÉRDIDAS DE AGUA Y SUELO POR EROSIÓN HÍDRICA
}

\section{EFFECT OF COVER CROPS ON WATER AND SOIL LOSSES DUE TO HYDRIC EROSION}

\author{
Julia Capurro $^{(1)(*)}$ y Sergio Montico ${ }^{(2)}$ \\ ${ }^{(1)}$ Agencia de Extensión Rural. Instituto Nacional de Tecnología Agropecuaria. Cañada de Gómez, Santa Fe, Argentina. \\ ${ }^{(2)}$ Facultad de Ciencias Agrarias, Universidad Nacional de Rosario. Zavalla, Santa Fe, Argentina. \\ ${ }^{(*)}$ e-mail: capurro.julia@inta.gob.ar
}

\begin{abstract}
RESUMEN
El objetivo de este trabajo fue evaluar el efecto de la inclusión de un cultivo de cobertura (CC) en una secuencia soja-soja, sobre las pérdidas de agua y suelo producidas por erosión hídrica en distintos ambientes de un suelo erosionado. El experimento se desarrolló sobre un suelo Argiudol típico, con un diseño de parcelas divididas, donde las parcelas mayores fueron los ambientes del sitio experimental, diferenciados por su relieve en: Loma (pendiente media de $0.15 \%$ ), Media Loma (pendiente media de $0.9 \%$ ) y Bajo (pendiente media $<0.1 \%$ ) y las parcelas menores correspondieron al subfactor tratamiento, con dos niveles: CCC (con cultivos de cobertura) y SCC (sin cultivos de cobertura) en base a una mezcla de avena (Avena sativa L.) y vicia (Vicia sativa L.). Se obtuvo la producción de materia seca (MS) de los CC y se aplicaron lluvias simuladas en cada parcela, a una intensidad constante de $60 \mathrm{~mm} / \mathrm{h}$ y una duración de 1 h, en prefloración del cultivo de soja sucesor. En cada parcela, después de la lluvia simulada, se registró la pérdida total de agua en $\mathrm{mm}\left(\mathrm{l} . \mathrm{m}^{2}\right)$ y se calculó el porcentaje respectivo sobre la lluvia total aplicada. Previamente a la aplicación de las lluvias simuladas, se evaluaron la cobertura vegetal (\%) y la rugosidad superficial en mm (RR). Se midió la pérdida total de suelo para cada lluvia en $\mathrm{g} / \mathrm{m}^{2}$. La cobertura superficial y la RR aumentaron significativamente por la inclusión de los CC. Las pérdidas totales de agua por escurrimiento superficial y de suelo en Loma, Media Loma y Bajo CCC fueron significativamente menores a las pérdidas en SCC. Los procesos de erosión hídrica fueron mitigados por los CC a través del aumento de la cobertura y rugosidad superficiales.
\end{abstract}

Palabras clave: erosión hídrica, degradación de suelos, cultivos de cobertura, monocultivo de soja

\begin{abstract}
The objective of this work was to evaluate the effect of the inclusion of a cover crop (CC) in a soybean-soybean sequence, on the losses of water and soil produced by water erosion in different environments of an eroded soil. The experiment was developed on a typical Argiudol soil, with a divided plot design, where the largest plots were the environments of the experimental site, differentiated by their topography in: Hump (average slope 0.15\%), Half hump (average slope $0.9 \%$ ) and Low (mean slope $<0.1 \%$ ) and the smaller plots corresponded to the treatment sub-factor, with two levels: CCC (with cover crops) and SCC (without cover crops) based on a mixture oats (Avena sativa L.) and vicia (Vicia sativa L.). The dry matter production (DM) of the CC was obtained and simulated rains were applied in each plot, at a constant intensity of $60 \mathrm{~mm} / \mathrm{h}$ and a duration of $1 \mathrm{~h}$, in pre-flowering of the successor soybean crop. In each plot, after the simulated rain, the total loss of water in $\mathrm{mm}\left(1 . \mathrm{m}^{2}\right)$ was recorded and the respective percentage of the total applied rain was calculated. Before applying the simulated rains, the vegetation cover (\%) and the surface roughness in $\mathrm{mm}(\mathrm{RR})$ were evaluated. Total soil loss was measured for each rain in g. $\mathrm{m}^{-2}$. Surface coverage and RR increased significantly due to the inclusion of CC. The total water losses due to surface and soil runoff in Hump, Half hump and Low CCC were significantly lower in SCC. The water erosion processes were mitigated by the CC through the increase in surface coverage and roughness.
\end{abstract}

Keywords: water erosion, soil degradation, cover crops, soybean monoculture 


\section{INTRODUCCIÓN}

Desde principios de la década del 90 del siglo veinte, la producción agrícola argentina registró un avance sostenido con un crecimiento extraordinario de la superficie destinada al cultivo de soja en las regiones pampeana y extrapampeana. Esta situación se potenció con la rápida incorporación de la soja transgénica asociada al sistema de siembra directa, que posibilitó su cultivo en áreas marginales y en suelos con limitaciones para la agricultura convencional (Casas, 2007).

No obstante el favorable impacto de la siembra directa sobre los suelos, persisten algunos procesos de degradación como la erosión hídrica. Una de las causas fundamentales de la erosión hídrica en las tierras cultivadas es la acción de las precipitaciones directamente sobre el suelo y la susceptibilidad del mismo a deteriorarse por las lluvias. La pérdida de suelo producida por este proceso, va a depender de la combinación de la erosividad, definida como el potencial de las precipitaciones para erosionar el suelo, con la erodibilidad del mismo, o sea, su capacidad de resistir la acción de las precipitaciones (Wischmeier y Smith, 1978; Hudson, 1995).

Dado que la erosión es un proceso predominantemente de superficie, las condiciones físicas de la capa edáfica superficial -externas e internas- son las que determinarán las pérdidas totales de suelo y agua. Principalmente, esas condiciones dependerán del tipo de uso de la tierra y del manejo del suelo y los cultivos, los cuales producen diferentes grados de cobertura y rugosidad superficiales (Bagatini et al., 2011).

En una agricultura pampeana dominada principalmente por el cultivo de soja, los aportes de los residuos de cosecha de este cultivo son bajos en cantidad y relación C:N, por lo que su participación en las rotaciones agrícolas afectan la conservación de los contenidos de materia orgánica y el mantenimiento de adecuados niveles de cobertura (Ruffo, 2003 citado por Scianca et al., 2008).

Esta problemática se agrava en áreas onduladas, donde gran parte del agua de las lluvias de primavera escurre hacia las zonas bajas de los lotes, arrastrando el escaso residuo de cosecha remanente. Así, la soja sembrada a continuación, emerge en suelos casi descubiertos (Capurro et al., 2010).

La inclusión de un cultivo de cobertura (CC) durante el período entre la cosecha de soja en otoño hasta la implantación de la soja sucesora, a mediados de la primavera siguiente, permite mantener el suelo cubierto y producir un nuevo ingreso de residuos de cosecha al sistema. La fitomasa aérea producida por el CC permanece entera y anclada al suelo a través de sus raíces y de esta forma no es arrastrada por los escurrimientos superficiales, como sucede con los residuos de los cultivos de renta, los cuales son trozados y esparcidos por la cosechadora.

El uso de simuladores de lluvia para el estudio de las relaciones lluvia-escorrentía, la medida de la tasa de infiltración y la estimación de las pérdidas de suelo, permiten experimentar estos procesos, controlando la intensidad, el sitio y la oportunidad de las precipitaciones.

El objetivo de este estudio fue evaluar el efecto de la inclusión de un CC en una secuencia soja-soja, sobre las pérdidas de agua y suelo producidas por erosión hídrica a través de la aplicación de lluvias simuladas, en distintos ambientes de un suelo erosionado perteneciente a un lote agrícola del sur de Santa Fe.

\section{MATERIALES Y MÉTODOS}

El estudio se realizó en un suelo Argiudol típico serie Correa, fase moderadamente erosionada (Horizonte superficial 0-16 cm: arcilla 23.5\%; arena $7.0 \%$; limo 69.5\%).

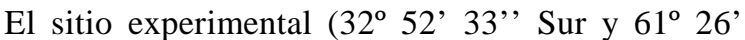
59 ''Oeste, altitud media de $114 \mathrm{~m}$ ) tuvo agricultura continua durante 25 años y monocultivo de soja de primera en siembra directa, durante los últimos diez años.

El experimento se realizó siguiendo un diseño en parcelas divididas. Las parcelas mayores correspondieron al factor ambiente con tres niveles: Loma (ambiente con relieve subnormal, con pendiente media de $0.15 \%$ y sin erosión hídrica), Media Loma (ambiente de relieve normal, con una pendiente media de $0.9 \%$, con erosión moderada y una pérdida de hasta el $25 \%$ del horizonte superficial) y Bajo (ambiente subnormal a cóncavo -pendiente media $<0.1 \%$ - con recepción y acumulación de sedimentos y sin erosión hídrica) y las parcelas menores correspondieron al subfactor tratamiento, con dos niveles: CCC (con cultivos de cobertura) y SCC (sin cultivos de cobertura). Las seis combinaciones de ambientes y tratamientos fueron: Loma CCC, Loma SCC, Media Loma CCC, Media Loma SCC, Bajo CCC y Bajo 
SCC. Las dimensiones de cada parcela resultaron de 30 $\mathrm{m}$ por $50 \mathrm{~m}$ y se distribuyeron en bloques completos aleatorizados con tres repeticiones. Se implantó una mezcla de avena (Avena sativa L.) -peso de 1000 semillas: 33 g- y vicia (Vicia sativa L.) -peso de 1000 semillas: 59 g- con una densidad de siembra de 88 $\mathrm{kg} / \mathrm{ha}$, a fin de lograr 200 plantas por metro cuadrado y una relación de consociación 50:50 de cada especie. La siembra del CC se realizó el 8 de mayo con una sembradora de siembra directa de grano fino, con líneas de siembra separadas a $0.175 \mathrm{~m}$ y abresurcos de doble disco plano. El ciclo de crecimiento del CC fue de 155 días, desde la emergencia hasta la supresión química de su crecimiento, el 13 de octubre. Al momento de la supresión química del crecimiento de los CC, la producción de materia seca (MS) se obtuvo mediante seis muestras de $0.25 \mathrm{~m}^{2}$ de la parte aérea de CC en cada parcela. Se llevó a estufa a $65{ }^{\circ} \mathrm{C}$ hasta peso constante, y se calculó la producción de MS en kg/ha. La siembra del cultivo de soja sucesor se efectuó el 10 de noviembre con una sembradora de siembra directa de grano grueso, con líneas de siembra separadas a $0.525 \mathrm{~m}$, cuchillas turbo, doble disco plantador y placas monograno.

Del 10 al 14 de diciembre, previo a la floración del cultivo de soja, se aplicó una lluvia simulada en cada parcela con un Simulador de Intensidades Múltiples, que cuantifica el proceso de erosión hídrica (Meyer y Harmon, 1979; Meyer, 1983), adaptado en la EEA INTA Marcos Juárez (Marelli et al., 1986). Previamente a la aplicación de las lluvias simuladas, se evaluó el porcentaje de cobertura vegetal de la superficie del suelo, por el método de la transecta lineal de Laflen et al. (1981) y la rugosidad superficial en $\mathrm{mm}$ (RR), utilizando un perfilómetro de agujas (Irurtia et al., 2010). Las lluvias simuladas fueron aplicadas a una intensidad constante de $60 \mathrm{~mm} / \mathrm{h}$ y tuvieron una duración de 1 h. Se instaló una microparcela de medición en cada unidad experimental. La micro-parcela de medición tuvo un área de $1 \mathrm{~m}^{2}(1 \mathrm{~m} \mathrm{x}$ $1 \mathrm{~m})$, con una zona de borde de $0.5 \mathrm{~m}$ de ancho y estuvo ubicada en la parte central del área de aspersión. Se delimitaron tres de sus lados con chapas galvanizadas de $20 \mathrm{~cm}$ de altura, enterradas $10 \mathrm{~cm}$ en el suelo y en el borde inferior, perpendicular a la dirección de la pendiente, se usó una bandeja aforadora del escurrimiento del mismo material, en forma de vertedero.

Se registró en cada lluvia simulada, la pérdida total de agua en mm (l. $\left.\mathrm{m}^{2}\right)$ y se calculó el porcentaje respectivo sobre la lluvia total aplicada durante la hora de duración del evento. Se midió la pérdida total de suelo para cada lluvia en $\mathrm{g} / \mathrm{m}^{2}$. Los sedimentos totales obtenidos de los escurrimientos se secaron a $105^{\circ} \mathrm{C}$ hasta peso constante y se convirtieron a kg/ha.

Los análisis estadísticos se realizaron mediante análisis de variancia según el diseño utilizado -parcelas divididas-, testeando particularmente los efectos de ambientes, tratamientos y su interacción. Seguidamente se realizaron los test de comparaciones de medias, de acuerdo a la significación de los efectos en los análisis de variancia, utilizando el test LSD de Fisher, a un nivel de significación del 5\%.

\section{RESULTADOS Y DISCUSIÓN}

Con el propósito de mostrar las condiciones ambientales que se presentaron durante el periodo del ensayo, se indica que la temperatura media fue de $12.93^{\circ} \mathrm{C}$, se registraron 19 heladas y las precipitaciones fueron de $391 \mathrm{~mm}, 50 \%$ por encima de la media histórica (1978/2011) para el período considerado. En el mes de diciembre -cuando se efectuó la medición de la lluvia artificial- la precipitación fue de 261mm.

La producción media de materia seca de los CC al momento del secado y en los diferentes ambientes fue: Loma: 5517 kg/ha; Media Loma: 5602 kg/ha; Bajo: $6734 \mathrm{~kg} / \mathrm{ha}$. No se hallaron diferencias significativas entre tratamientos y tampoco entre ambientes. La cobertura superficial fue significativamente superior en Loma CCC, Media Loma CCC y Bajo CCC (93\%, $91 \%$ y $97 \%$, respectivamente), en comparación con Loma SCC, Media Loma SCC y Bajo SCC (38\%, 27\% y 50\%). Asimismo, la RR mostró diferencias significativas a favor de Loma CCC, Media Loma CCC y Bajo CCC (17.2; 19.7 y 16.4 mm, respectivamente) en relación a Loma SCC, Media Loma SCC y Bajo SCC (6.5; 2.9 y $7.8 \mathrm{~mm}$, respectivamente).

\section{Efecto en las pérdidas totales de agua}

Las pérdidas totales de agua por escurrimiento superficial (Figura 1), mostraron efectos de tratamientos y ambientes altamente significativos, sin interacción entre ambos. Los registros fueron menores en las parcelas CCC con una media de $24 \%$ de agua total perdida, mientras que en las parcelas SCC los valores fueron de $38 \%$ en promedio. En Loma, Media Loma y Bajo, las pérdidas medias fueron de $27 \%$, $38 \%$ y $27 \%$, respectivamente. 


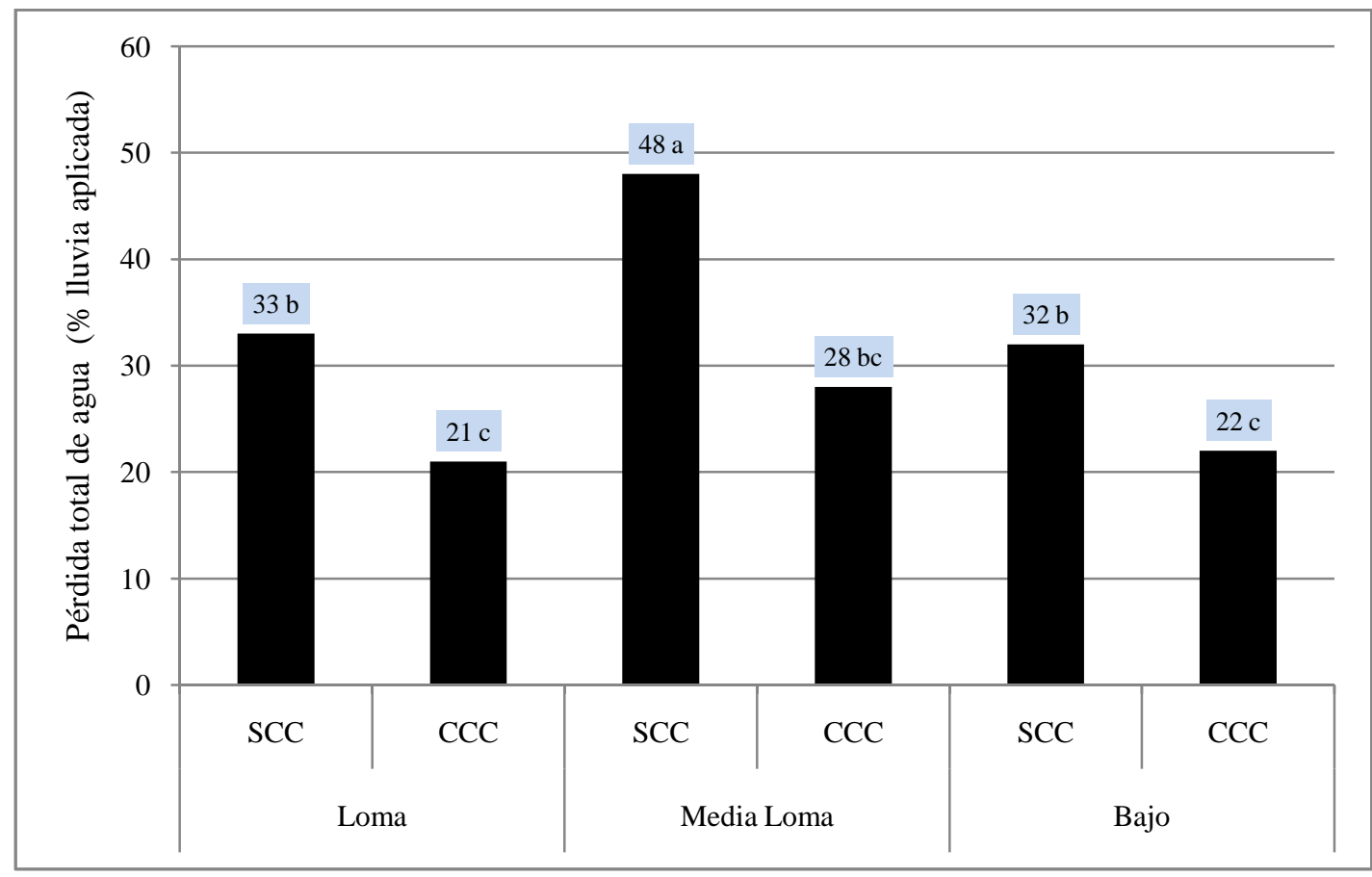

Figura 1. Pérdida total de agua por escurrimiento superficial, en porcentaje sobre la lluvia aplicada.

Ref: SCC:Sin Cultivos de Cobertura; CCC: Con Cultivos de Cobertura. Letras distintas indican diferencias significativas (p < 0.05).

La significativa disminución de los escurrimientos superficiales, en las parcelas CCC respecto de las SCC en cada ambiente, pudo generarse por el efecto de la cobertura vegetal, asociado al efecto de una más alta rugosidad. Estas condiciones, posiblemente, aumentaron la capacidad del suelo de resistir la acción erosiva de la lluvia, al impedir el contacto directo de las gotas con la superficie, e incrementaron la retención superficial del agua, mejorando su infiltración en el suelo. Coincidiendo con esto, Crespo et al. (2010) aplicaron lluvias simuladas en 34 sitios del centro de la provincia de Buenos Aires y determinaron que, independientemente del sistema de labranza utilizado, el escurrimiento dependió en gran parte de la cobertura vegetal de los mismos.

Las mayores diferencias establecidas entre los tratamientos en la Media Loma, pudieron estar relacionadas con el relativamente alto gradiente de la pendiente $(0.9 \%)$, en un suelo con muy baja rugosidad debido a las lluvias anteriores $\mathrm{y}$ densificado superficialmente, en oportunidad de la siembra de soja después de un largo período de años en siembra directa. Estos factores contribuyeron al escurrimiento de una mayor masa de agua en un breve período de tiempo, provocando una pérdida equivalente a la mitad de la lluvia aplicada en las parcelas SCC.
Diversos autores (Bertol, 1986; Levien, 1999; Volk, 2002; Streck y Cogo, 2003; Cogo y Streck, 2003; Volk et al., 2004 y Castro et al., 2006, citados por Gilles et al., 2009) han observado que la escasa remoción del suelo en la siembra directa -dado por la profundidad de labor, la extensión de superficie de terreno trabajado y el nivel de fragmentación del volumen de suelo removido- ocasiona una disminución de la rugosidad superficial y ningún incremento adicional de porosidad total del horizonte superficial edáfico, además de resultar en una superficie de suelo generalmente consolidada. Esta condición, según los autores, favorece con el tiempo la conservación del suelo, pero en muchos casos, desfavorece la conservación del agua, por el aumento del escurrimiento superficial.

En este trabajo, el relieve en Loma y Bajo, probablemente, no favoreció la dinámica del agua en la misma proporción que en Media Loma, por lo que las condiciones de cobertura y rugosidad no fueron afectadas en la misma medida, no obstante alrededor de un tercio del agua aplicada se perdió por escurrimientos superficiales. En este sentido, Bertol y Miquelluti (1993) evaluaron pérdidas por erosión hídrica con distintos manejos de suelo y observaron que las pérdidas de agua fueron mucho menos influenciadas por los sistemas de manejo del suelo que las pérdidas de suelo, lo que condice, según estos autores, con la mayoría de los trabajos conducidos en ese área. 
También con lluvias simuladas, Gilles et al. (2009) encontraron pérdidas de agua por escurrimientos superficiales, de hasta $71 \%$ y $46 \%$ de la lluvia aplicada en los momentos de siembra y floración, respectivamente, del cultivo de maíz (Zea mays) en siembra directa sobre suelos Ultisoles. Con resultados diferentes sobre una situación productiva contrastante, Bagatini et al. (2011) hallaron pérdidas de agua de menos del 20\% de la lluvia aplicada, en un suelo con pocos años de agricultura y en base a un manejo conservacionista de labranzas y fertilización.

\section{Efecto en las pérdidas totales de suelo}

Se observaron efectos altamente significativos de tratamiento y ambiente, sin interacción entre ambos. Las parcelas CCC (Figura 2) tuvieron pérdidas de suelo significativamente menores a las parcelas SCC, con una media de $131 \mathrm{~kg} / \mathrm{ha}$ en contraste con $524 \mathrm{~kg} / \mathrm{ha}$ de promedio en las últimas. A su vez, el ambiente Media Loma mostró pérdidas de suelo significativamente superiores a los ambientes Loma y Bajo, con medias de 603, 244 y 136 kg/ha, respectivamente.

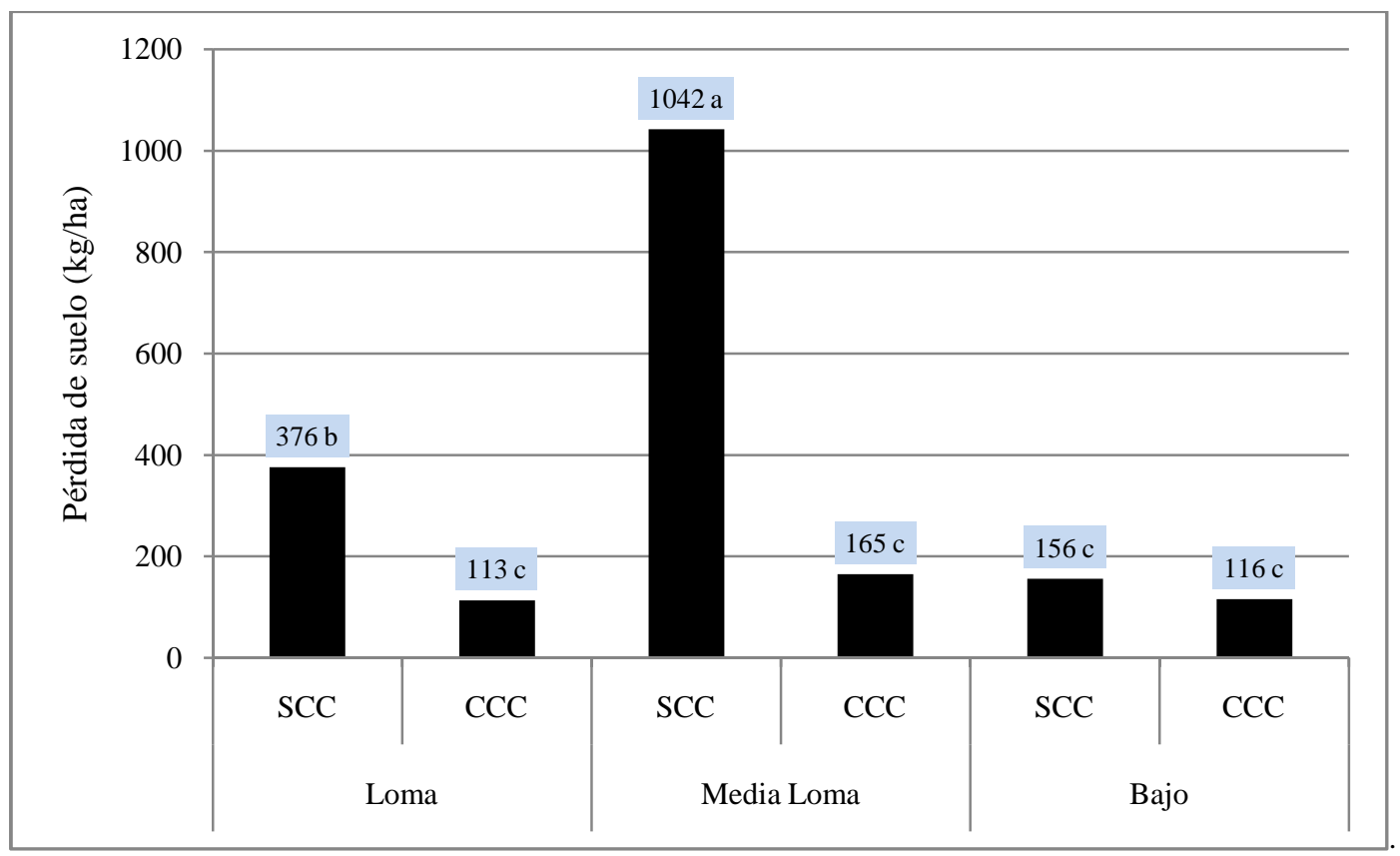

Figura 2. Pérdida total de suelo en kilos por hectárea, por erosión hídrica.

Ref: SCC: Sin Cultivos de Cobertura; CCC: Con Cultivos de Cobertura. Letras distintas indican diferencias significativas(p < 0.05).

El efecto de los CC fue de gran magnitud en la reducción de las pérdidas de suelo en Loma y Media Loma, probablemente debido a la protección frente al efecto erosivo de la lluvia aplicada, así como a la acción mecánica de las raíces, con diferencias entre ambientes en función de su posición en el relieve. Las gotas pueden llegar a ser muy erosivas cuando golpean un suelo sin vegetación, de acuerdo a Morgan (1997) citado por De la Cruz (2010), proporcionando además de la fuerza disgregadora, una fuerza de consolidación que lo compacta. El impacto es el agente más importante para el desprendimiento y disgregación de las partículas (De la Cruz, 2010) y la mayor parte de la energía que llevan las gotas precipitadas se va a consumir en dicho proceso.

Según Portela et al. (2011), el proceso de transporte de las partículas de suelo erosionadas por el agua generalmente es más alterado que el escurrimiento superficial, respecto a las condiciones externas de la superficie del suelo. Weir (2002), trabajando con parcelas de escurrimiento con $1 \%$ de pendiente, determinó que el escurrimiento fue cinco veces mayor y la pérdida de suelo doce veces superior en las parcelas con suelo descubierto, que el promedio de parcelas cultivadas y con pasturas. A su vez, Bertol y Miquelluti (1993) citados por Torres et al. (2005) encontraron que las pérdidas de suelo y agua se reducían en un $85 \%$ y $29 \%$, respectivamente cuando incorporaron cultivos de cobertura. Barcelonna y Rienzi (2003) encontraron disminuciones de 70\% de la pérdida total de suelo al aplicar cobertura sobre un suelo con labranza convencional y $25 \%$ al aplicarla sobre un suelo proveniente de pradera.

No hubo diferencias significativas en la pérdida de 
suelo entre tratamientos en el Bajo posiblemente debido a que, si bien las parcelas CCC mostraron los valores más altos de cobertura, también las SCC presentaron valores elevados. Esto podría estar vinculado con la acumulación de residuos de cosecha de soja provenientes de los ambientes superiores, desplazados por los movimientos de agua en superficie, y pudo influir en su respuesta ante la lluvia aplicada, disminuyendo las pérdidas de suelo en las parcelas sin CC de este ambiente.

\section{CONCLUSIONES}

Hubo un elevado incremento de la rugosidad y cobertura superficial del suelo por la inclusión de los CC. Los procesos de erosión hídrica fueron mitigados en todos los ambientes que tuvieron la cubierta vegetal invernal. Las pérdidas de agua por erosión hídrica, fueron menores en las parcelas CCC y lo mismo sucedió con las pérdidas de suelo. En el ambiente Media Loma, por sus características topográficas, las diferencias en estos parámetros fueron de mayor magnitud.

\section{REFERENCIAS}

Bagatini, T.; Cogo, N. P.; Gilles, L.; Portela, J. C.; Portz, P. y Queiroz, H.T. (2011). Perdas de solo e água por erosão hídrica após mudança no tipo de uso da terra, em dois métodos de preparo do solo e dois tipos de adubação. Revista Brasileira de Ciência do Solo, 35, 999-1011.

Barcelonna, C. y Rienzi, E. A. (2003). Cambios producidos por la cobertura sobre la relación de enriquecimiento del sedimento erosionado en un Argiudol típico. Revista Facultad de Agronomia, 23 (2-3), 141-145.

Bertol, I. (1986). Relações da erosão hídrica com métodos de preparo do solo, na ausência e na presença de cobertura vegetal por resíduos culturais de trigo. (Tese de Mestrado. Universidade Federal do Rio Grande do Sul, Porto Alegre).

Bertol, I. y Miquelluti, D. J. (1993). Perdas de solo, agua e nutrientes reduzidas pela cultura do milho. Pesquisa Agropecuária Brasileira, 28(10), 12051213.

Capurro, J.; Surjack, J.; Andriani, J.; Dickie, M. J. y Gonzalez, M. C. (2010). Evaluación de distintas especies de cultivos de cobertura en secuencias sojasoja en el área sur de la provincia de Santa Fe. p.1-5 (Resumen expandido). XXII Congreso Argentino de la Ciencia del Suelo. Rosario, Santa Fe.

Casas, R. (2007). Preservar la calidad y salud de los suelos: una oportunidad para la Argentina. Anales de la Academia Nacional de Agronomía y Veterinaria, LX, 37-61.

Castro, L. G.; Cogo, N. P. y Volk, L. B. S. (2006) Alterações na rugosidade superficial do solo pelo preparo e pela chuva e sua relação com a erosão hídrica. Revista Brasileira de Ciência do Solo, 30, 339-352.

Cogo, N. P. y Streck, E. V. (2003) Surface and subsurface decomposition of a desiccated grass pasture biomass related to erosion and its prediction with RUSLE. Revista Brasileira de Ciência do Solo, 27, 153-164.

Crespo, R. J.; Sfeir, A.; Usunoff, E.; Ares, G. y Wingeyer, A. B. (2010). Efecto de la labranza y la cobertura vegetal sobre el escurrimiento y la pérdida de suelo en la Región central de la provincia de Buenos Aires. Revista de la Facultad de Ciencias Agrarias de la Universidad Nacional de Cuyo, 42 (1), 93-106.

De La Cruz, I. (2010). Caracterización de lluvia simulada y su aplicación en parcelas experimentales de erosión en taludes de infraestructuras lineales. (Trabajo Fin de Carrera. Escuela Universitaria de Ingeniería Técnica Forestal. Universidad Politécnica de Madrid).

Gilles, L.; Cogo, N. P.; Bissani, C. A.; Bagatini, T. y Portela, J. C. (2009). Perdas de água, solo, matéria orgânica e nutriente por erosão hídrica na cultura do milho implantada em área de campo nativo, influenciadas por métodos de preparo do solo e tipos de adubação. Seção VI - manejo e conservação do solo e da água. Revista Brasileira de Ciência do Solo, 33, 1427-1440.

Hudson, N. W. (1995). Soil conservation. ( $3^{\text {ra }}$.ed.) Ames, lowa State University Press.

Irurtia, C. B.; Mon, R.; Gonzalez, N.; Elisei, J.; Cruzate, G. y A. Llovet. (2010). Variación en el corto plazo del microrelieve y la cobertura superficial en un suelo subsolado bajo siembra directa. p. 1-4 (Resumen expandido). XXII Congreso Argentino de la Ciencia del Suelo. Rosario, Santa Fe.

Laflen, J. M.; Amemiya, M. y Hintz, E.A. (1981). Measuring crop residue cover. Journal of Soil and Water Conservation, 36, 341-343.

Levien, R. (1999). Condições de cobertura $e$ métodos de preparo do solo para a implantação da cultura do milho (ZeamaysL.). (Tese de Doutorado. Universidade Estadual Paulista, Botucatu).

Marelli, H. J.; Arce, J. M. y Massiero, B.L. (1986). 
Simulador de lluvias para investigación básica en conservación de suelos. Publicación Técnica Nº8. Serie Suelos y Agroclimatología. EEA INTA Marcos Juárez.

Meyer, L. (1983). Portable rainfall simulator. Comunicación personal.

Meyer, L.D. y Harmond, W.C. (1979). Multiple Intensity Rainfall Simulator for erosion research on row side slopes. Transactions of the ASAE. 22 (1): 0100-0103. doi: 10.13031/2013.34973. Morgan, R. P. C. (1997). Erosión y conservación del suelo. Ediciones Mundi-Prensa.

Ruffo, M. (2003). Factibilidad de inclusión de cultivos de cobertura en Argentina. Actas XI Congreso de AAPRESID, pp. 171-176.

Portela, J. C.; Cogo, N. P.; Do Amaral, A. J.; Gilles, L.; Bagatini, T.; Pardo Chagas, J. y Portz, G. (2011). Hidrogramas e Sedimentogramas associados à erosão hídrica em solo cultivado com diferentes sequências culturais, com diferentes condições físicas na superfície. Revista Brasileira de Ciência do Solo, 35, 225-240.

Scianca, C.; Álvarez, C.; Barraco, M.; Quiroga, A. y M. B. Pérez. (2008). Impacto de diferentes coberturas invernales sobre propiedades edáficas, población de malezas y productividad de soja. Memoria Técnica 2007-08. EEA INTA General Villegas.

Streck, E.V. y Cogo, N. P. (2003). Reconsolidation of the soil surface after tillage discontinuity, with and without cultivation, related to erosion and its prediction with RUSLE. Revista Brasileira de Ciência do Solo, 27,141-152.

Torres R., D.; Florentino, A. y López, M. (2005). Pérdidas de suelo y nitrógeno por escorrentía en un Ultisol degradado bajo diferentes condiciones de cobertura vegetal en Chaguaramas-Guárico. Agronomía Tropical, 55(4), 475-496.
Volk, L. B. S. (2002). Erosão hídrica relacionada às condições físicas de superfície e subsuperfície do solo, induzidas por formas de cultivo e de manejo dos resíduos culturais. (Tese de Mestrado. Universidade Federal do Rio Grande do Sul, Porto Alegre)

Volk, L. B. S.; Cogo, N. P. y Streck, E. V. (2004). Erosão hídrica influenciada por condições físicas de superfície e subsuperfície do solo resultantes do seu manejo, na ausência de cobertura vegetal. Revista Brasileira de Ciência do Solo, 28, 763-774.

Weir. E. (2002). Pérdida de suelo y agua en parcelas de escurrimiento. p.1-7. (Informe Técnico) Actas del Segundo Taller de Contaminación por Agroquímicos, 23 Agosto 2002. Pergamino. Buenos Aires.

Wischmeier, W. H. y Smith, D. D. (1978). Predicting rainfall erosion losses; a guide to conservation planning. Agricultural Handbook, 537. Washington, USDA.

\section{Tipo de Publicación: NOTA TÉCNICA.} Trabajo recibido el 03/04/2020 y aprobado para su
publicación el 01/08/2020.

\section{COMO CITAR}

Capurro, J. y Montico, S. (2020). Efecto de los cultivos de cobertura sobre las pérdidas de agua y suelo por erosión hídrica. Cuadernos del CURIHAM. 26:41-47. DOI: https://doi.org/10.35305/curiham.v26i0.147

Este es un artículo de acceso abierto bajo licencia: Creative Commons Atribución - No Comercial Compartir Igual 4.0 Internacional (CC BY-NC-SA 4.0) (https://creativecommons.org/licenses/by-ncsa/4.0/deed.es) 